\title{
ROCKing pulmonary fibrosis
}

\author{
Dean Sheppard, MD
}

Lung Biology Center, Department of Medicine, UCSF, San Francisco, California, USA.

\begin{abstract}
Clinical vignette: A 76-year-old man consults you for increasing shortness of breath over the past two years and an increasing requirement for home oxygen. A video-assisted thoracoscopic lung biopsy shows findings of usual interstitial pneumonitis, and he has no identifiable cause for pulmonary fibrosis, so he is considered to have idiopathic pulmonary fibrosis (IPF). His diffusing capacity for carbon monoxide (DLCO) is $45 \%$ of predicted, and his total lung capacity is $40 \%$ of predicted. Because of his advanced age, he is not considered a candidate for lung transplantation. What treatment should you recommend?
\end{abstract}

\section{Current therapy}

Idiopathic pulmonary fibrosis (IPF) is a devastating lung disease with no currently approved effective therapies. The median survival after diagnosis is approximately three years. For decades, patients with this disease were treated with a variety of cytotoxic and immunosuppressive drugs, but it is now clear that these treatments do more harm than good. Progressive, fatal pulmonary fibrosis also occurs as a consequence of numerous other diseases, including hypersensitivity pneumonitis, rheumatoid arthritis, and scleroderma. The outcome of pulmonary fibrosis in these diseases is not as uniformly grim, and some patients who are not severely affected improve after antigen removal (in the case of hypersensitivity pneumonitis) or immunosuppressive drug treatment. However, once patients with these diseases develop moderately severe fibrosis and significant impairment of lung function, most have a very high risk of progression and death, with little evidence of beneficial effect of pharmacologic intervention. These clinical characteristics indicate that significant pulmonary fibrosis, whatever the initiating events, might itself predispose patients to progression of the fibrotic process - suggesting the potential existence of one or more feedforward circuits through which tissue fibrosis begets more fibrosis.

Conflict of interest: The author is co-owner of patents for treatment of pulmonary fibrosis with antibodies blocking the $\alpha v \beta 6$ integrin and has a sponsored research agreement with Biogen/Idec to study the efficacy of anti-integrin antibodies for treatment of tissue fibrosis.

Citation for this article: J Clin Invest. 2013; 123(3):1005-1006. doi:10.1172/JCI68417.

\section{Knowledge gap}

Recent work from a number of experimental systems has implicated tissue stiffness as an important determinant of multiple cell behaviors. In one of the most striking examples, mesenchymal stem cells plated onto soft substrates differentiate into brain cells, cells plated on substrates of intermediate stiffness differentiate into muscle, and cells plated on the stiffest substrates differentiate into bone (1). These findings suggest that cells must have precise mechanisms to detect and appropriately respond to changes in the stiffness of their immediate surroundings. Previous work has identified examples of this phenomenon of "mechanotransduction" that are relevant to tissue fibrosis. For example, lung fibroblasts plated on artificial soft substrates are resistant to differentiation into myofibroblasts and make substantially less collagen than fibroblasts plated on artificial stiff substrates (2). Furthermore, stiff substrates have been shown to facilitate cellular activation of latent TGF- $\beta$ through integrins expressed on fibroblasts or epithelial cells $(3,4)$. The recently solved crystal structure of the latent complex of TGF- $\beta$ provided a compelling explanation of how mechanical force applied through integrins by contracting fibroblasts or epithelial cells could cause release of the active TGF- $\beta$ cytokine from the embrace of its own carboxyterminal fragment, and how tissue stiffness likely facilitates this process (5). These findings indicate that increased tissue stiffness in the lungs could contribute to fibrosis.

\section{Research advances}

In this issue of the JCI, Zhou et al. provide new insight into how fibrosis, once established, can beget more fibrosis (6).
They describe a pathway through which tissue stiffness, in the setting of pulmonary fibrosis, promotes the differentiation and survival of pulmonary myofibroblasts. This pathway requires the activity of Rho-associated kinases (ROCK), which is necessary for contraction and force generation in fibroblasts and for downstream actin polymerization. The authors demonstrate that ROCK-induced actin polymerization leads to nuclear translocation of the transcriptional coactivator, MKL1, which itself plays important roles in promoting the differentiation and survival of myofibroblasts. Enhanced survival is mediated at least in part by induction of the well-characterized survival factor B cell lymphoma 2 (BCL-2). These results suggest several steps that could be targeted to break the feed-forward circuit connecting tissue stiffness with fibrosis progression.

One of the most exciting possibilities, which was directly tested by Zhou et al. (6), is that pulmonary fibrosis could be treated by inhibition of ROCK. Several potent ROCK inhibitors are already available, and one of these, fasudil, has been approved for clinical use in Japan and China and has already been given to a large number of patients for prevention of vasoconstriction after brain injury. Another ROCK inhibitor, Y-27932, was previously shown to prevent pulmonary fibrosis in response to bleomycin (7), but in that study, the drug was administered before bleomycin and might have protected against acute lung injury and inflammation. These steps are important in the bleomycin model, but less relevant to patients with ongoing fibrosis. Zhou et al. showed that fasudil could prevent fibrosis even when administered 14 days after bleomycin (6), at which time inflammation has largely resolved and fibrosis is already present.

ROCK inhibitors such as fasudil could be effective for treating pulmonary fibrosis by more than one mechanism. These drugs have been previously shown to prevent the contraction of lung epithelial cells that is required for activation of TGF- $\beta$ by the epithelial $\alpha v \beta 6$ integrin. 
ROCK-dependent fibroblast contraction has also been shown to be important for TGF- $\beta$ activation by other $\alpha v$-containing integrins present on myofibroblasts, which link mechanotransduction to feedforward circuits that promote fibrosis progression. By targeting multiple parallel feed-forward circuits, ROCK inhibitors could be especially promising as antifibrotic drugs.

\section{Implications and future directions}

One important caveat could limit the utility of ROCK inhibitors for treatment of fibrotic diseases. ROCK activity is critical for contraction of vascular smooth muscle and thus contributes to the homeostatic vascular smooth muscle contraction required for the maintenance of normal blood pressure. Depending on the relative degrees of ROCK inhibition required to prevent pathologic tissue fibrosis and relax vascular smooth muscle, the utility of systemically administered ROCK inhibitors could be limited by the induction of hypotension. One strategy that deserves further consideration for the treatment of pulmonary fibrosis is chemical modification of ROCK inhibitors to slow absorption into the bloodstream and increase systemic metabolism - modifications that could enrich for pulmonary effects if the drugs are administered by aerosol directly into the lung. Such a strategy might allow effective treatment of pulmonary fibrosis while minimizing the risk of systemic toxicity.

\section{Acknowledgments}

The author is supported by NHLBI grants HL108794, HL102292, and HL53949; by NIAID grant AI077439; and by a grant from the Scleroderma Research Foundation.

Address correspondence to: Dean Sheppard, UCSF MC 2922, San Francisco, California
94143-2922, USA. Phone: 415.514.4269; Fax: 415.514.4278; E-mail: Dean.Sheppard@ ucsf.edu.

1. Engler AJ, Sen S, Sweeney HL, Discher DE. Matrix elasticity directs stem cell lineage specification. Cell. 2006;126(4):677-689.

2. Liu F, et al. Feedback amplification of fibrosis through matrix stiffening and COX-2 suppression. J Cell Biol. 2010;190(4):693-706.

3. Giacomini MM, Travis MA, Kudo M, Sheppard D. Epithelial cells utilize cortical actin/myosin to activate latent TGF-beta through integrin alpha(v) beta(6)-dependent physical force. Exp Cell Res. 2012;318(6):716-722.

4. Wipff PJ, Rifkin DB, Meister JJ, Hinz B. Myofibroblast contraction activates latent TGFbeta1 from the extracellular matrix. J Cell Biol. 2007;179(6):1311-1323.

5. Shi M, et al. Latent TGF-beta structure and activation. Nature. 2011;474(7351):343-349.

6. Zhou Y, et al. Inhibition of mechanosensitive signaling in myofibroblasts ameliorates experimental pulmonary fibrosis. J Clin Invest. 2013; 123(3):1096-1108.

7. Shimizu Y, et al. Contribution of small GTPase Rho and its target protein rock in a murine model of lung fibrosis. Am J Respir Crit Care Med. 2001;163(1):210-217. 